\title{
Application of conjugate gradient method for estimation of the wall heat flux of a supersonic combustor
}

\author{
Liuwei Cheng, Fengquan Zhong*, Hongbin Gu, Xinyu Zhang \\ State Key Laboratory of High Temperature Gas Dynamics, Institute of Mechanics, Chinese Academy of Sciences, No. 15 Beisihuanxi Road, Beijing 100190, China
}

\section{A R T I C L E I N F O}

\section{Article history:}

Received 5 June 2015

Received in revised form 12 January 2016

Accepted 12 January 2016

Available online 29 January 2016

\section{Keywords:}

Conjugate gradient method

Inverse heat conduction problem

Supersonic combustor

Wall heat flux

\begin{abstract}
A B S T R A C T
In this paper, conjugate gradient method (CGM) considering variations in material properties with temperature is developed to solve transient inverse heat conduction problems. With CGM, the measured outer wall temperatures of supersonic combustor are used as input data to recover the heat flux and temperature on the inner wall. Numerical test has been done to study the effect of temperature measurement noises on accuracy of the inversion result. It indicates that the inversion results are very close to the exact heat flux with a maximum error of less than $5 \%$ when the measurement noise is less than $\pm 3 \mathrm{~K}$. At the same time, a series of experiments are conducted on a Mach 3 supersonic combustor test facility with varied fuel injection conditions. The heat fluxes on the inner wall are recovered by measured outer wall temperatures via CGM. The inversion results of heat flux agree satisfactorily with the values measured by heat flux sensors and a maximum difference of less than $5 \%$ is found. The present comparison results prove the validity and accuracy of the CGM developed in the paper for estimation of the wall heat flux of supersonic combustor.
\end{abstract}

(C) 2016 Elsevier Ltd. All rights reserved.

\section{Introduction}

Heat flux on the combustor inner wall is one of the most critical parameters for design and optimization of thermal protection of engine combustors. Both direct and indirect measurement techniques have been developed to measure the inner wall heat flux and temperature. Heat flux sensor, directly contacting the combustor flow, is the most commonly used apparatus [1,2]. However, its operation lifetime and reliability are limited due to combustor flow with high temperature, high speed and considerable fraction of oxygen. Additionally, because of its relatively large size especially with cooling structure, the spatial resolution of heat flux sensor is usually low and its response time is as large as a few seconds or more [3]. The indirect measurements include optical method, of which the inner wall temperature can be determined by infrared radiation of the heated wall surface [4,5]. However, intense emission from combustion flame and reflected light from other wall surfaces would cause significant errors in the measured wall temperature.

In this paper, time change of temperature on the outer wall of supersonic combustor is measured and used as the input data to determine the inner wall heat flux and temperature by conjugate

\footnotetext{
* Corresponding author.

E-mail address: fzhong@imech.ac.cn (F. Zhong).
}

gradient method (CGM) based on the principle of heat conduction through solid wall and calculus of variations. Oliveria and Orlande [6] evaluated surface heat flux of ablative material using CGM. Mohammadium et al. [7] studied accuracy and numerical stability of CGM when the measured temperatures (as the input data) had noises $[8,9]$. However, most of the previous conjugate gradient methods are implemented with the assumption that thermal properties of wall material such as thermal conductivity or specific heat are constant [7-9]. It is known that for gas turbine engine or ramjet/scramjet combustor, temperature across the combustor wall can vary from room temperature on the outer wall to more than $1000 \mathrm{~K}$ on the inner wall due to large heat flux with magnitudes of $\mathrm{MW} / \mathrm{m}^{2}[10,11]$. The significant change in the wall temperature leads to large variations in thermal properties of wall material. Therefore, significant error in the recovered inner wall heat flux would exist if wall thermal parameters are still regard as constant in the inversion analysis. More importantly, most of the previous applications of CGM are for heat transfer analysis of reentry vehicles. To the authors' knowledge, application of CGM for thermal measurements of supersonic combustor has not been reported yet.

In the present study, CGM considering variations in material properties of the combustor wall is developed and the accuracy of the inversion results is examined when the input temperature has noises with different values. Using CGM, the measured outer-wall temperatures of a Mach 3.0 supersonic combustor via 
thermocouples or infrared measurements are used to determine the inner wall heat flux. The recovered inner wall heat fluxes are compared to the results obtained with commercial hightemperature heat flux sensors and good agreements between them are found. The present study proves feasibility and reliability of the present CGM for thermal measurements of supersonic combustor.

\section{Description of the conjugate gradient method}

A three-dimensional object of solid wall with heat loading is shown in Fig. 1. The initial temperature of the object is uniform and equals $T_{0}$. The heat flux $q_{w}(0, y, z, t)$ on the upper surface is a function of time as well as $y$ and $z$ distances. Four side-wall surfaces are assumed to be adiabatic since heat flux component in the direction vertical to the combustor wall is much larger than that in the direction perpendicular to the wall. The lower surface, representing the outer wall of combustor, is also considered to be adiabatic since heat transfer through the outer wall is dominated by natural convection and the heat flux value is negligible with comparison to the inner wall heat flux. With CGM, the measured temperature on the lower surface $T(L, y, z, t)$ is used as the input data to determine the heat flux on the upper surface.

The conjugate gradient method is used to solve unconstrained optimization problems such as inverse heat conduction problem described in Section 2.2. The purpose of CGM is to find a heat flux function on the upper surface that leads to a temperature distribution on the lower surface closest to the measured one. The problem has been transferred to a minimization problem of functional (Eq. (2) in Section 2.2). Assuming an initial guess of heat flux, the final function can be searched by Eq. (3a) in Section 2.3 in which the search direction can be obtained by gradient of functional as described in Section 2.5 and the search step size can be determined by solving sensitivity equation in Section 2.4.

\subsection{Direct heat conduction problem}

Formulation of direct heat conduction problem is given as follows:

$\rho c \frac{\partial T}{\partial t}=\frac{\partial}{\partial x}\left[k(T) \frac{\partial T}{\partial x}\right]+\frac{\partial}{\partial y}\left[k(T) \frac{\partial T}{\partial y}\right]+\frac{\partial}{\partial z}\left[k(T) \frac{\partial T}{\partial z}\right]$

for $0<x<L, 0<y<M, 0<z<N, t>0$

$-k(T) \frac{\partial T}{\partial x}=q(y, z, t) \quad$ for $\quad x=0, t>0$

$-k(T) \frac{\partial T}{\partial x}=0$ for $x=L, t>0$

$-k(T) \frac{\partial T}{\partial y}=0$ for $y=0, M, t>0$

$-k(T) \frac{\partial T}{\partial z}=0$ for $z=0, N, t>0$

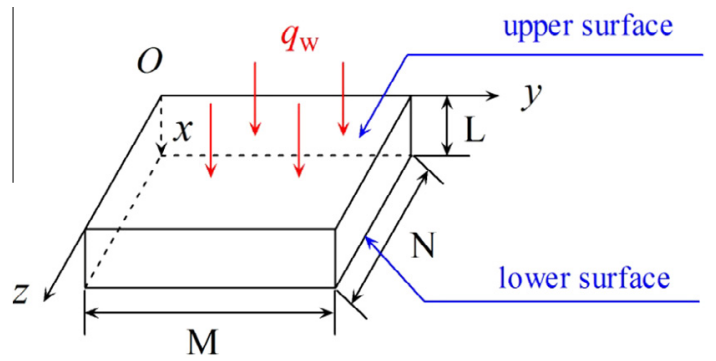

Fig. 1. Schematic diagram of the 3-dimensional wall with heating loading.
$T=T_{0} \quad$ for $\quad 0 \leqslant x \leqslant L, 0 \leqslant y \leqslant M, 0 \leqslant z \leqslant N, t=0$

In the direct problem, heat flux on the upper surface is known and distribution of wall temperature at arbitrary time is calculated from Eq. (1) with 2nd-order finite difference scheme.

\subsection{Inverse heat conduction problem}

For the inverse problem, heat flux on the upper surface can be recovered by using the temperature on the lower surface. In view of high sampling rate of temperature measurement, the change of temperature can be regard as a continuous function of time. Therefore, the inverse problem can be solved by minimizing the following functional:

$$
\begin{aligned}
J[q(y, z, t)] & =\int_{0}^{t_{f}} \sum_{i=1}^{i_{m}}\left[T\left(x_{i}, y_{i}, z_{i}, t\right)-Y\left(x_{i}, y_{i}, z_{i}, t\right)\right]^{2} d t \\
& =\int_{0}^{t_{f}} \sum_{i=1}^{i_{m}}\left[T_{i}(t)-Y_{i}(t)\right]^{2} d t
\end{aligned}
$$

where, $T$ and $Y$ denote the calculated and the measured temperature on the lower surface respectively. The subscript " $i$ " is $i$ th moment during the measurement period.

\subsection{Conjugate gradient method}

The essential idea of CGM is that the minimization problem of the above functional (2) can be transformed into solutions of the direct problem of heat conduction, the sensitivity equation and the adjoint problem [11-15]. Hence, heat flux on the upper surface satisfying the temperature change on the lower surface can be obtained by the iterative process as described as follows:

$q^{n+1}(y, z, t)=q^{n}(y, z, t)-\beta^{n} P^{n}(y, z, t)$ for $n=0,1,2, \ldots$

where $\beta^{n}$ is the search step size from iteration $n$ to $n+1$. And $P^{n}$ is the search direction given by the following conjugate equation:

$P^{n}(y, z, t)=J^{\prime n}(y, z, t)+\gamma^{n} P^{n-1}(y, z, t)$

where, $J^{n}$ as called as the gradient direction, denotes derivative of the functional $J[q(y, z, t)]$ for time and for $y$ and $z$ distances and it is determined by solving the adjoint equation as described in Section 2.5. The conjugate coefficient $\gamma^{n}$ is determined from:

$\gamma^{n}=\frac{\int_{0}^{t_{f}} \sum_{i=0}^{i_{m}}\left[J^{\prime n}\left(y_{i}, z_{i}, t\right)\right]^{2} d t}{\int_{0}^{t_{f}} \sum_{i=0}^{i_{m}}\left[J^{\prime n}\left(y_{i}, z_{i}, t\right)\right]^{2} d t} \quad$ with $\quad \gamma^{0}=0$

To perform the iterative process given by Eq. (3a), the search step size $\beta^{n}$ and search direction $P^{n}$ are needed. To obtain the two parameters, a sensitivity problem and an adjoint problem are constructed in the following.

\subsection{Sensitivity problem and search step size}

Sensitivity problem can be obtained by the limiting approach as described in literature [16]. When $q$ is perturbed by $\Delta q$, temperature $T$ undergoes a variation of $\Delta T$. Plugging $q+\Delta q$ and $T+\Delta T$ into Eq. (1), subtracting the Eq. (1) and neglecting the second-order terms, the sensitivity equation (i.e. variation equation) can be obtained as follows:

$$
\begin{aligned}
& \rho c \frac{\partial \Delta T}{\partial t}=\frac{\partial^{2}(k \Delta T)}{\partial x^{2}}+\frac{\partial^{2}(k \Delta T)}{\partial y^{2}}+\frac{\partial^{2}(k \Delta T)}{\partial z^{2}} \\
& \text { for } 0<x<L, 0<y<M, 0<z<N, t>0 \\
& -\frac{\partial(k \Delta T)}{\partial x}=\Delta q(y, z, t) \text { for } x=0, t>0
\end{aligned}
$$


$-\frac{\partial(k \Delta T)}{\partial x}=0$ for $x=L, t>0$

$-\frac{\partial(k \Delta T)}{\partial y}=0$ for $y=0, M ; t>0$

$-\frac{\partial(k \Delta T)}{\partial z}=0$ for $z=0, N ; t>0$

$\Delta T=0 \quad$ for $\quad 0 \leq x \leq L, 0 \leq y \leq M, 0 \leq z \leq N, t=0$

After solving the sensitivity equation, the search step size $\beta^{n}$ can be determined by Eq. (5) as follows:

$\beta^{n}=\frac{\int_{0}^{t_{f}} \sum_{i=0}^{i_{m}}\left[T_{i}(t)-Y_{i}(t)\right] \Delta T_{i}(t) d t}{\int_{0}^{t_{f}} \sum_{i=0}^{i_{m}}\left[\Delta T_{i}(t)\right]^{2} d t}$

\subsection{Adjoint problem and gradient equation}

To obtain the adjoint problem, multiplying Eq. (1a) by Lagrange multiplier $\lambda$ and integrating the result over the time and spatial domain and then adding the integration result to the right-handside of Eq. (2), the functional $J$ can be rewritten as:

$$
\begin{aligned}
J[q(y, z, t)]= & \int_{0}^{t_{f}} \sum_{i-1}^{i_{m}}\left[T_{i}(t)-Y_{i}(t)\right]^{2} d t+\int_{0}^{t_{f}} \int_{\Omega}\left\{\frac{\partial}{\partial x}\left[k(T) \frac{\partial T}{\partial x}\right]\right. \\
& \left.+\frac{\partial}{\partial y}\left[k(T) \frac{\partial T}{\partial y}\right]+\frac{\partial}{\partial z}\left[k(T) \frac{\partial T}{\partial z}\right]-\rho c \frac{\partial T}{\partial t}\right\} \lambda d x d y d z d t
\end{aligned}
$$

With limiting process of Eq. (6), the adjoint problem can be obtained:

$$
\begin{aligned}
& \rho c \frac{\partial \lambda}{\partial t}+\frac{\partial^{2} \lambda}{\partial x^{2}}+\frac{\partial^{2} \lambda}{\partial y^{2}}+\frac{\partial^{2} \lambda}{\partial z^{2}}=0 \\
& \text { for } 0<x<L, 0<y<M, 0<z<N, t>0 \\
& -k \frac{\partial \lambda}{\partial x}=0 \text { for } x=0, t>0 \\
& -k \frac{\partial \lambda}{\partial x}=-2(T-Y) \delta\left(x-x_{i}\right) \delta\left(y-y_{i}\right) \delta\left(z-z_{i}\right) \text { for } x=L, t>0 \\
& -k \frac{\partial \lambda}{\partial y}=0 \text { for } y=0, M, t>0 \\
& -k \frac{\partial \lambda}{\partial z}=0 \text { for } z=0, N, t>0 \\
& \lambda=0 \text { for } 0 \leq x \leq L, 0 \leq y \leq M, 0 \leq z \leq N, t=t_{f}
\end{aligned}
$$

where $\delta(\cdot)$ is the Dirac $\delta$ function and $t_{f}$ is the total time. Eq. (6) can be simplified as:

$\Delta J[q(y, z, t)]=\int_{z=0}^{N} \int_{y=0}^{M} \int_{0}^{t_{f}} \lambda(0, y, z, t) \cdot \Delta q(y, z, t) d t d y d z$

The variation of functional can be written as:

$\Delta J[q(y, z, t)]=\int_{z=0}^{N} \int_{y=0}^{M} \int_{0}^{t_{f}} J^{\prime}[q(y, z, t)] \cdot \Delta q(y, z, t) d t d y d z$

A comparison of Eqs. (8a) and (8b) leads to the result for the gradient of functional:

$J^{\prime}[q(y, z, t)]=\lambda(0, y, z, t)$

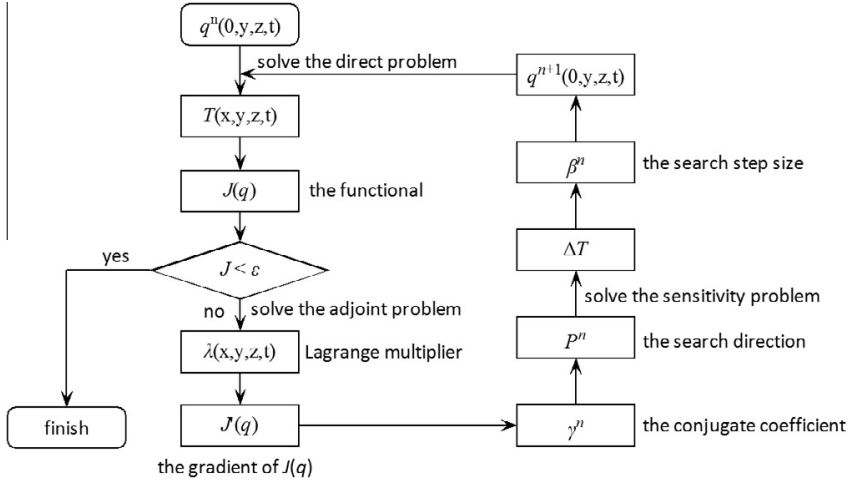

Fig. 2. The solution procedure of CGM.

\subsection{Convergence criterion}

If the measurement data (the input data) contains no errors or noises, the convergence criterion of iteration is as follows:

$J[q(y, z, t)]<\varepsilon)$

where $\varepsilon$ is a small-specified number. In actual experiment, measurement errors is unavoidable and it can be expressed as follows:

$T_{i}(t)-Y_{i}(t) \approx \sigma$

where $\sigma$ is the stand deviation of measurements. Substituting Eq. (10b) into Eq. (2), the following expression for $\varepsilon$ is obtained:

$\varepsilon=i_{m} \sigma^{2} t_{f}$

where, $i_{m}$ is the total number of temperature measurement points.

\subsection{Computational procedure}

The solution procedure of inverse heat conduction problem using CGM is summarized by a flow chart as given in Fig. 2 .

\section{Influence of measurement noise}

To analyze the effect of measurement noises of input temperature on the inversion result, a three-dimensional heat conduction problem is considered. As shown in Fig. 1, the distance between the upper and the lower surfaces is $4 \mathrm{~mm}$, the size of the upper and the lower surfaces is $16 \mathrm{~mm} \times 16 \mathrm{~mm}$. The solid-wall material is stainless steel, which has an initial uniform temperature of $300 \mathrm{~K}$ and a density of $7930 \mathrm{~kg} / \mathrm{m}^{3}$. The specific heat of stainless steel can be described as a linear function of temperature $C_{p}=362.295$ $+0.377 T$ in unit of $J /(\mathrm{kg} \mathrm{K})$ and the heat conduction coefficient is described as $k=12.2+0.011 T$ in unit of $\mathrm{W} /(\mathrm{m} \mathrm{K})$. A distribution of heat flux as functions of time and $y$ and $z$ distances $\left(q_{w}=\exp \left[-\frac{(y-M / 2)^{2}+(z-N / 2)^{2}}{20}\right](1+0.1 t)\right.$ in unit of $\left.\mathrm{MW} / \mathrm{m}^{2}\right)$ is imposed on the upper surface and the time change of temperature on the lower surface is used as input data. The heat flux and wall temperature on the upper surface are recovered by using CGM with a time step of $0.01 \mathrm{~s}$ and a mesh size of $0.5 \mathrm{~mm}$. In the procedure of CGM, a 2nd-order central scheme is applied for the spatial discretization and the temporal discretization is calculated by 2 ndorder explicit scheme.Contours of the exact wall heat flux on the upper surface at $t=4 \mathrm{~s}$ are shown in Fig. 3(a). The inversion results of the heat flux with input temperature noise of $\pm 1 \mathrm{~K}, \pm 3 \mathrm{~K}$ and $\pm 5 \mathrm{~K}$ are given in Fig. 3(b)-(d) respectively. As shown in Fig. 3, in overall, the three inversion results at varied temperature noises can recover distribution of the exact wall heat flux. As the input noise increases, the inversion value of maximum heat flux (at the center 


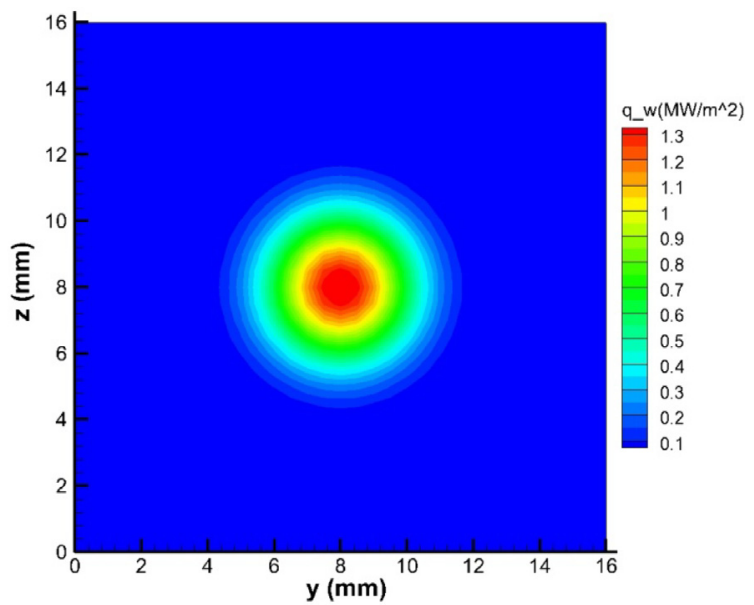

(a) The exact wall heat flux on the top wall at $t=4 \mathrm{~s}$

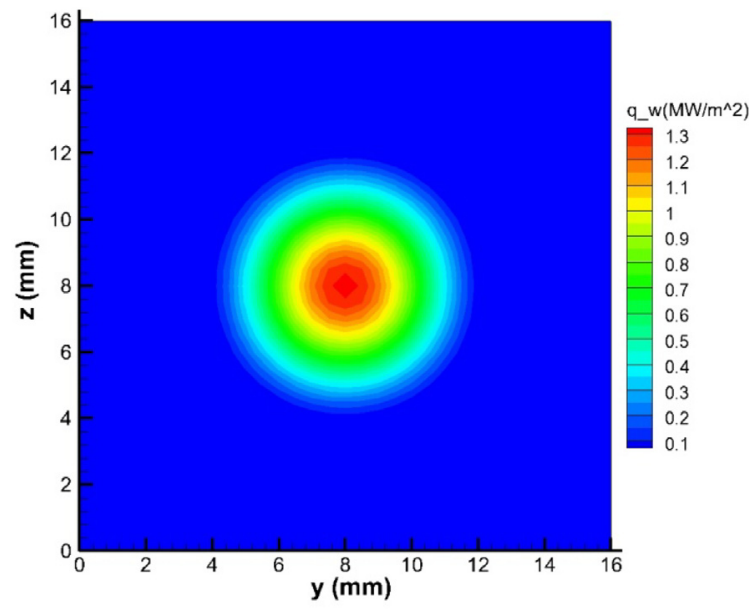

(c) The inversion result with a temperature noise of $\pm 3 \mathrm{~K}$

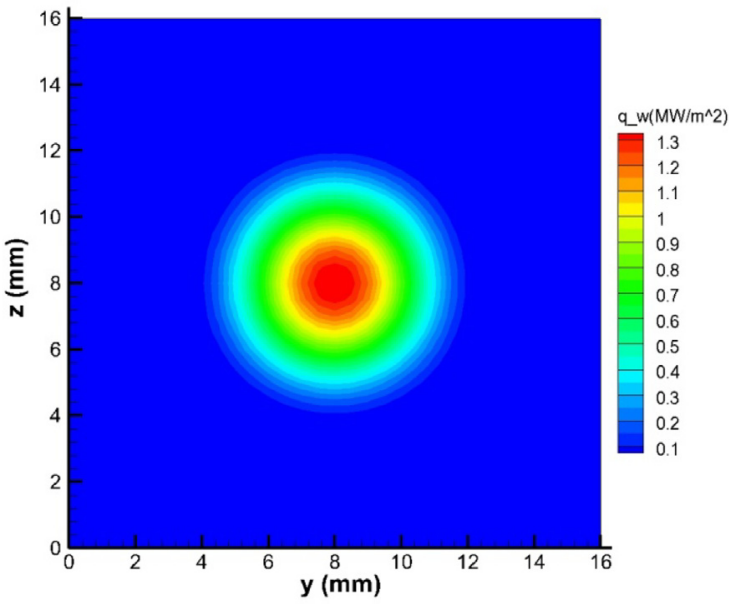

(b) The inversion result with a temperature noise of $\pm 1 \mathrm{~K}$

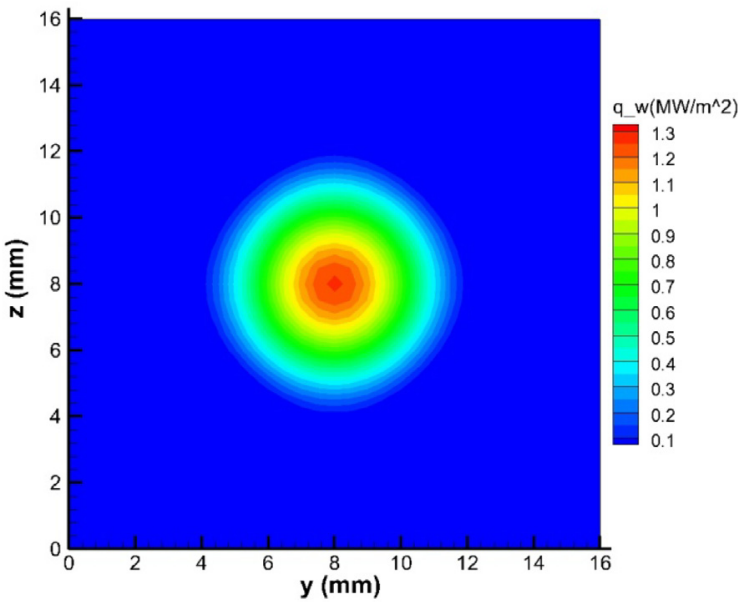

(d) The inversion results with a temperature noise of $\pm 5 \mathrm{~K}$

Fig. 3. The exact wall heat flux and inversion results.

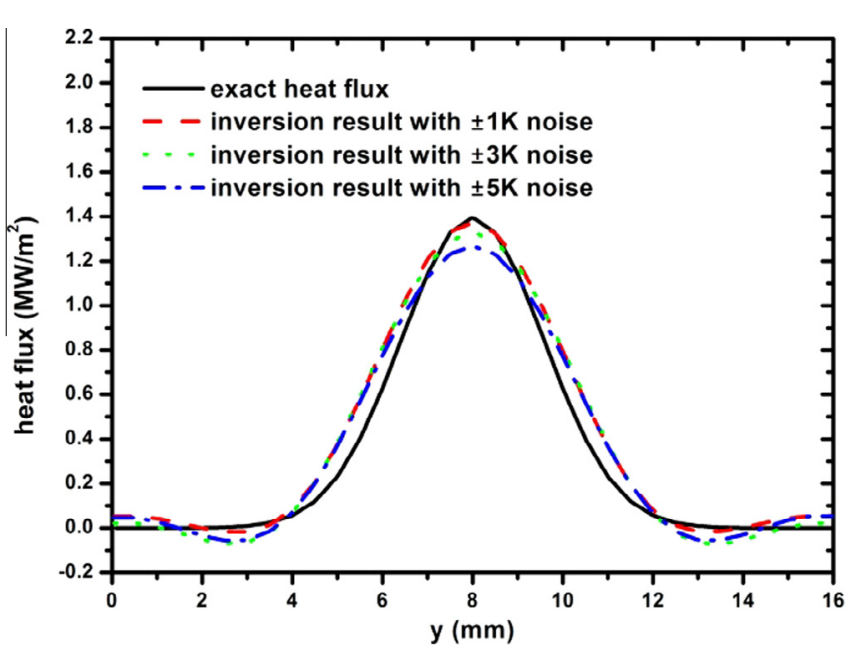

Fig. 4. The comparison of exact heat flux and inversion results with different noises.

of the upper surface) becomes smaller than the exact value. In order to show effect of measurement noise more clearly, a comparison of the exact heat flux and the inversion results at the line of $z=0.008 \mathrm{~m}$ is given in Fig. 4. As shown in the figure, difference between the exact heat flux and the inversion results is clearly observed and the error in the inversion heat flux increases with the noise. The relative error in the maximum heat flux of the upper surface is $2.1 \%, 5.0 \%$ and $9.2 \%$ for the noise of $\pm 1 \mathrm{~K}, \pm 3 \mathrm{~K}$ and $\pm 5 \mathrm{~K}$ respectively. Considering the measurement error of the outerwall temperature is usually about $\pm 1 \mathrm{~K}$ in the experiments, the CGM can be used to recover the actual inner-wall heat flux of combustor with sufficiently high accuracy.

\section{Experimental results}

Measurement of wall heat flux is conducted on a direct-connect supersonic combustor facility of which its geometry and flow parameters have been described in details in our previously published work [17]. The vitiated air flow is heated to a temperature of about $1650 \mathrm{~K}$ by hydrogen/air combustion and then accelerates to supersonic speed (Mach number 3 ) via sonic nozzle. A sketch of the facility with locations of wall temperature measurements (point 1,2 and region A) are shown in Fig. 5. The ethylene fuel is injected from multiple holes on the strut upstream of the cavity.

At location 1 and 2 as indicated in Fig. 5, two type-K thermocouples are spot-welded on the outer surface of the combustor side 


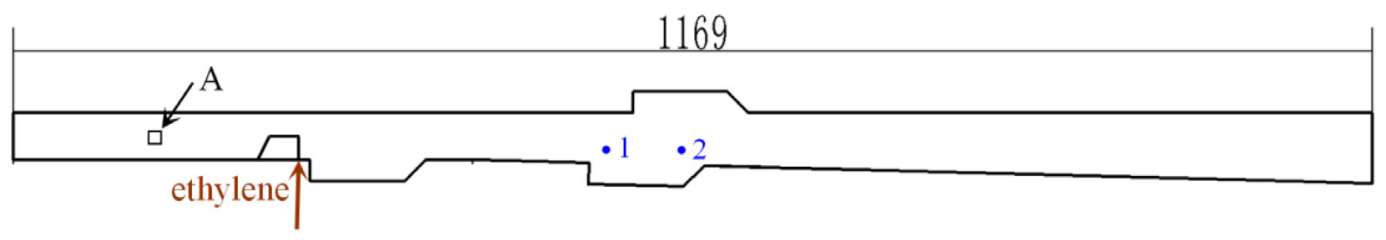

(a) Schematic diagram

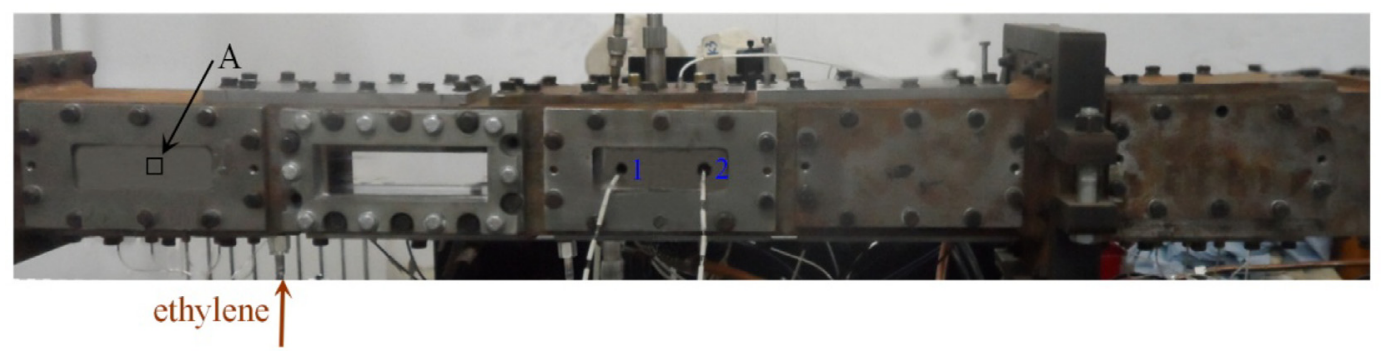

(b) Photograph of the facility

Fig. 5. Direct-connect test facility with heat flux measurements.

wall to measure the time change of the outer wall temperature for inversion of the inner wall heat flux. At the same downstream and vertical positions and on the opposite side wall, two hightemperature Gardon heat flux sensors are installed to directly measure the inner wall heat flux. Since wall heat flux on the two side walls should be nearly the same due to symmetry of combustor configurations and fuel injection, the values of wall heat flux obtained with thermocouples and heat flux sensors can be compared for validation of the CGM.

The inlet temperature and Mach number of the combustor is $1650 \mathrm{~K}$ and 3 respectively and the inlet mass flow rate is $1.62 \mathrm{~kg} /$ $\mathrm{s}$. The ethylene fuel is injected from a moment of $t=2.3 \mathrm{~s}$ after the supersonic flow has been well developed in the combustor. The fuel/air equivalence ratio is 0.43 and the fuel injection lasts for a period of two and a half seconds $(t=2.3-4.8 \mathrm{~s})$. Fig. 6 gives distribution of wall pressure along the axial direction at $t=4.3 \mathrm{~s}$ after fuel combustion has been stabilized. For comparison, wall pressure without combustion is also plotted and significant increases in the wall pressure is observed, that is due to heat releasing of combustion.

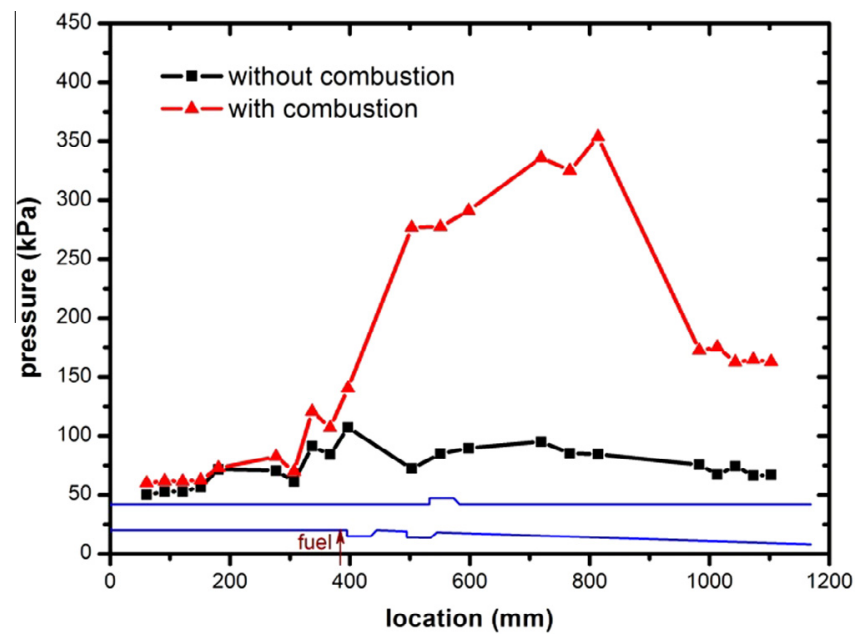

Fig. 6. Distribution of wall pressure along the axis direction.
Fig. 7 gives the time change of the outer wall temperature measured by thermocouples at location 1 and 2 . As the input data of CGM, the outer wall temperatures have measurement noises of less than $\pm 1 K$. Fig. 8 (a) and (b) plot the inversion results of wall heat flux at location 1 and 2 as well as the values directly measured by heat flux sensors. In the CGM process, the time step for the numerical solution of the heat conduction equation is set to be $0.01 \mathrm{~s}$ and the grid size in the vertical direction is $0.1 \mathrm{~mm}$.

As shown in the figures, overall, the inversion results of wall heat flux agree satisfactorily with those measured by heat flux sensors. The maximum wall heat flux, the most concerned parameter for thermal protection of combustor, is found to be very close to each other for the two methods and a maximum difference of less than $5 \%$ is found. However, some differences in the heat flux curves are clear in the figures that the inversion heat flux curves are smoother than the direct measurements by heat flux sensor. It attributes to the numerical dissipation of the finite difference scheme and relatively coarse meshes for solutions of the heat conduction equation (1a) and the sensitivity equation (4a).

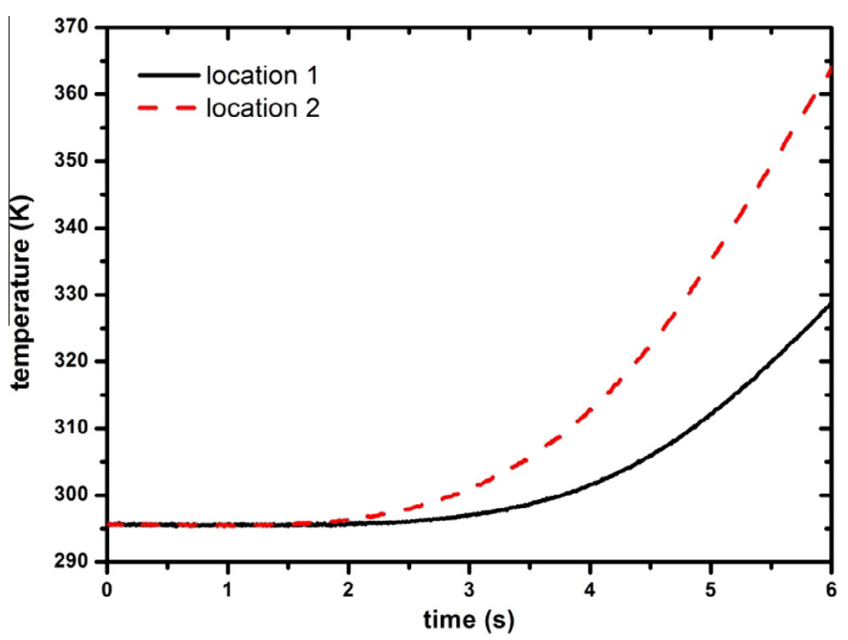

Fig. 7. Time change of the outer wall temperature measured by thermocouples 


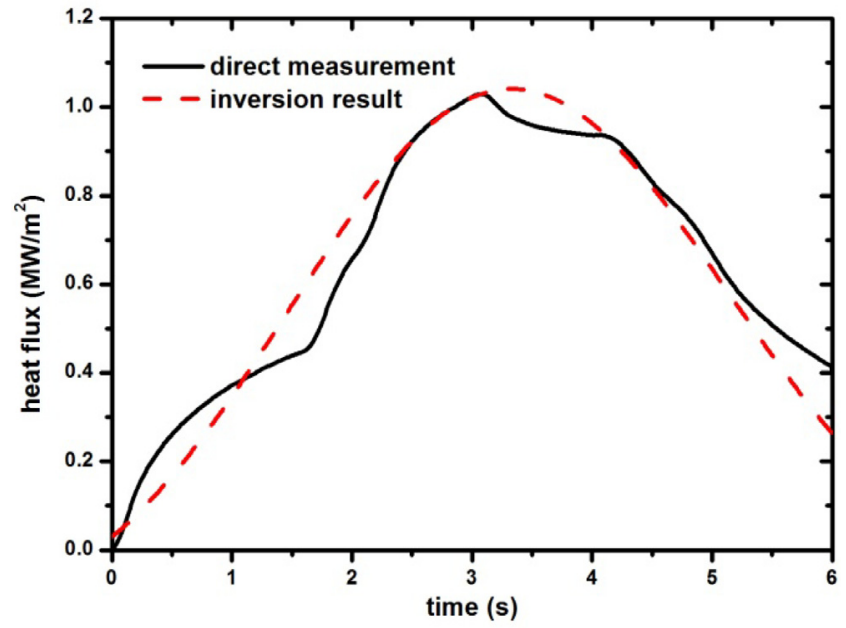

(a) Location 1

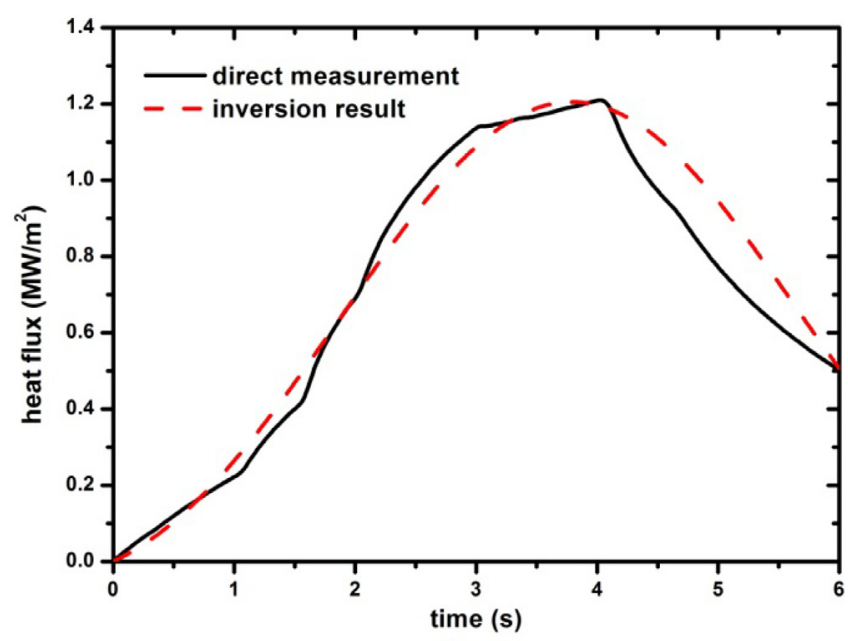

(b) Location 2

Fig. 8. Comparisons of inner wall heat flux directly measured by sensors and by CGM.

Table 1 summarizes the maximum wall heat flux obtained by the CGM and by the heat flux sensors at location 1 and 2 for different test cases with varied fuel/air equivalence ratios. It is found the difference between the inversion result and the directly measured value of maximum heat flux is less than $5 \%$ for all the test cases and locations. As fuel/air equivalence ratio increases, the wall heat flux increases due to larger heat releasing from fuel/air combustion. As shown in the table, for all the cases, the maximum heat flux at location 2 is higher than that at location 1 since location 2 is more downstream and more close to the cavity than location 1 . Therefore, combustion in the vicinity of location 2 is more intense than that near location 1.

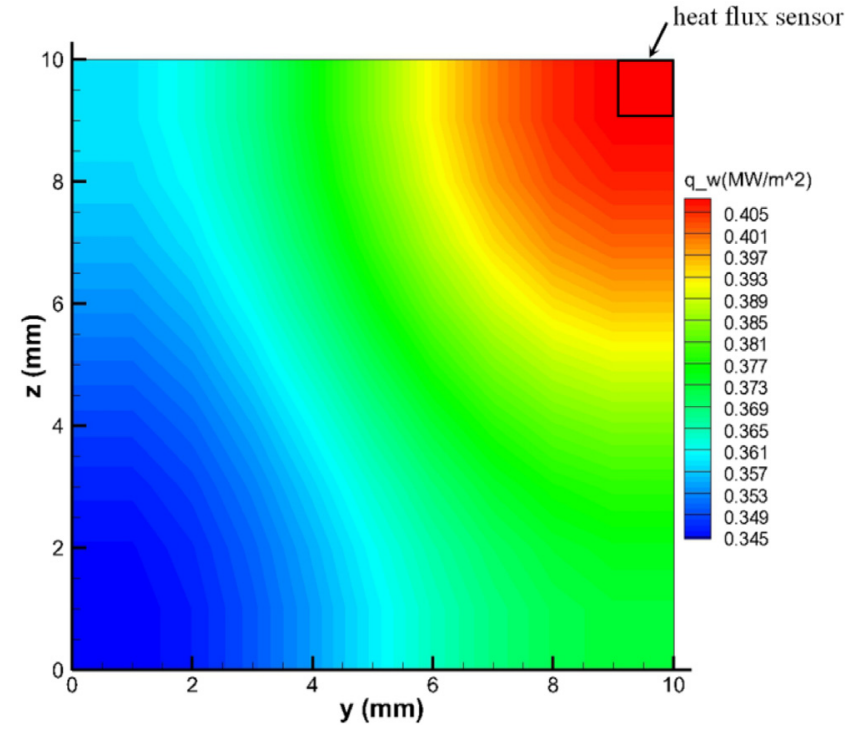

(a) $\mathrm{t}=3 \mathrm{~s}$

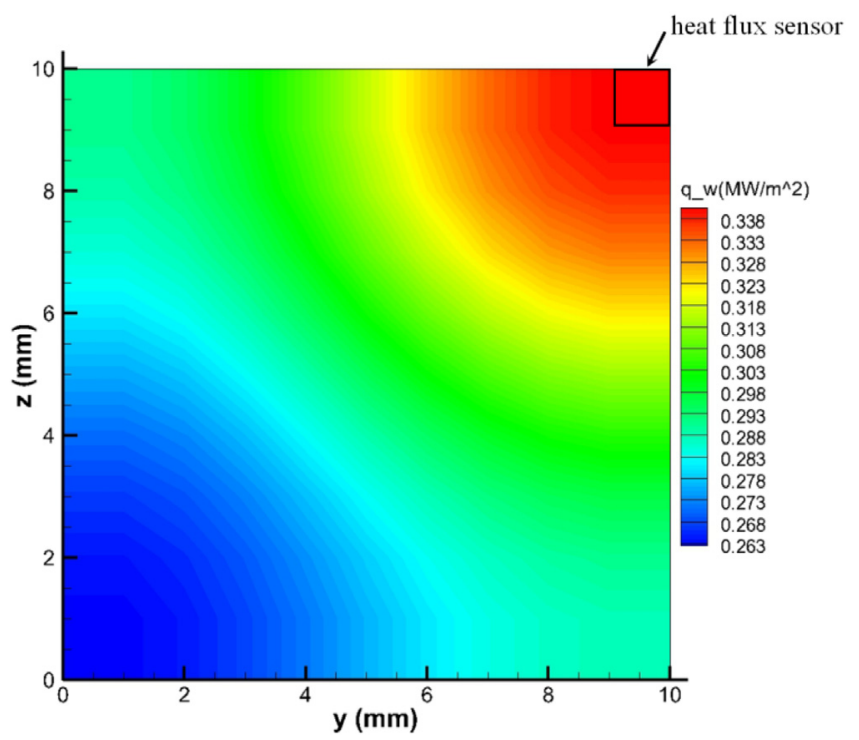

(b) $t=5 \mathrm{~s}$

Fig. 9. Inversion results of spatial distribution of the inner wall heat flux at varied times.

In order to obtain spatial distribution of the wall heat flux, infrared temperature measurement is conducted to obtain time evolution of the outer wall temperature in a specific region of $10 \mathrm{~mm} \times 10 \mathrm{~mm}$ in the isolator of the combustor as indicated by symbol "A" in Fig. 6(a). The infrared camera can measure wall surface temperature from $273 \mathrm{~K}$ to $800 \mathrm{~K}$ with an accuracy of $\pm 1 \mathrm{~K}$ and with a spatial resolution of $0.8 \mathrm{mrad}$. With two-dimensional distribution of the measured outer wall temperature, distribution of the

Table 1

Comparison of maximum wall heat flux obtained by heat flux sensor and by CGM.

\begin{tabular}{|c|c|c|c|c|c|c|}
\hline \multirow{2}{*}{$\begin{array}{l}\text { Fuel/air } \\
\text { equivalence ratio }\end{array}$} & \multicolumn{3}{|l|}{ Location 1} & \multicolumn{3}{|l|}{ Location 2} \\
\hline & $\begin{array}{l}\text { Direct measurement } \\
\left(\mathrm{MW} / \mathrm{m}^{2}\right)\end{array}$ & $\begin{array}{l}\text { Inversion result with CGM } \\
\left(\mathrm{MW} / \mathrm{m}^{2}\right)\end{array}$ & $\begin{array}{l}\text { Relative } \\
\text { error (\%) }\end{array}$ & $\begin{array}{l}\text { Direct measurement } \\
\left(\mathrm{MW} / \mathrm{m}^{2}\right)\end{array}$ & $\begin{array}{l}\text { Inversion result with CGM } \\
\left(\mathrm{MW} / \mathrm{m}^{2}\right)\end{array}$ & $\begin{array}{l}\text { Relative } \\
\text { error (\%) }\end{array}$ \\
\hline 0.43 & 1.03 & 1.01 & -1.9 & 1.21 & 1.19 & -1.7 \\
\hline 0.57 & 1.12 & 1.13 & 0.9 & 1.3 & 1.36 & 4.3 \\
\hline 0.61 & 1.18 & 1.21 & 2.5 & 1.45 & 1.49 & 2.8 \\
\hline
\end{tabular}


heat flux on the inner wall can be obtained by CGM. Fig. 9 gives contours of inner wall heat flux in the region of A at different moments. The spatial distribution and the local peak of inner wall heat flux can be clearly identified.

As shown in Fig. 9, a Gardon heat flux sensor is installed at the right-up corner of region A on the opposite side wall to directly measure the inner wall heat flux. Compared to the direct measured heat flux, the relative error of the inversion result is $-4.23 \%$ and $-3.40 \%$ for time of $t=3 \mathrm{~s}$ and $t=5 \mathrm{~s}$ respectively. It again proves that the CGM developed in the paper can be used to obtain the inner wall heat flux with high spatial resolutions and good accuracy.

\section{Conclusion}

In this paper, a three-dimensional inverse heat conduction problem is solved by conjugate gradient method (CGM), and distribution of heat flux on the inner wall of combustor is recovered by the outer wall temperatures with CGM. With consideration of variations in thermal parameters of the wall material with temperature, the CGM developed in the present study is able to obtain inner wall thermal properties of supersonic combustor having significant temperature difference across the wall thickness. Numerical study indicates that the CGM can give accurate inversion result of wall heat flux (with relative errors less than 5\%) when the noise of the input outer wall temperature is less than $\pm 3 \mathrm{~K}$. Feasibility and accuracy of the CGM is also investigated by combustion and heat transfer experiments on the direct-connect supersonic combustor facility. The inversion results of wall heat flux are found to agree satisfactorily well with that measured by Gardon sensors and a maximum difference of less than $5 \%$ is achieved. In addition, infrared temperature measurement with the CGM is used to obtain a spatial distribution of heat flux on the inner wall of isolator of the combustor. And the recovered heat flux field on the inner wall again proves the present CGM can be used to obtain inner wall heat flux with high spatial resolutions and satisfactory accuracy.

\section{Acknowledgment}

This work is funded by Natural Science Foundation of China under Contract No. 11172309 and 91441102.

\section{References}

[1] Long Li, Jing Wang, Xuejun Fan, Development of integrated high temperature sensor for simultaneous measurement of wall heat flux and temperature, Rev. Sci. Instrum. 83 (2012) 074901.

[2] Paul J. Kennedy, Jeffrey M. Donbar, Heat flux measurements in a scramjet combustor using direct write technology, AIAA paper 2011-2330, 2011.

[3] T. Tsuru, S. Tomioka, K. Kudo, A. Murakami, K. Kato, H. Yamasaki, Skin-friction measurements in supersonic combustion flows of a scramjet combustor, AIAA Paper 2008-4578, 2008

[4] Xinxian Dang, Jianxing Zhao, Yingwen Yan, Yong Liu, Rong Xu, Xin Zhang, Experiment of wall temperature in a two-stage swirler combustor, J. Aerosp. Power 25 (2010) 1227-1232.

[5] D. Liu, Y.Y. Duan, Z. Yang, Effects of participating media on the time-resolved infrared measurement of wall temperature in a coal-fired combustor, Exp. Thermal Fluid Sci. 39 (2012) 90-97.

[6] Alexandre P. DE Oliveira, Helcio R.B. Orlande, Estimation of the heat flux at the surface of ablating materials by using temperature and surface position measurements, Inverse Prob. Sci. Eng. 12 (5) (2004) 563-577.

[7] M. Mohammadiun, A.B. Rahimi, I. Khazaee, Estimation of the time-dependent heat flux using the temperature distribution at a point by conjugate gradient method, Int. J. Therm. Sci. 50 (2011) 2443-2450.

[8] Ching-yu Yang, Chao'o-Kuang Chen, The boundary estimation in twodimensional inverse heat conduction problems, J. Phys. D: Appl. Phys. 29 (1996) 333-339.

[9] Alemdar Hasanov, Identification of space wise and time dependent source terms in 1D heat conduction equation from temperature measurement at a final time, Int. J. Heat Mass Transfer 55 (2012) 2069-2080.

[10] D. Suslov, K. Dullenkopf, A. Schulz, S. Wittig, Development and application of a novel technique for direct heat flux measurements in turbomachinery flows, Flow Turbul. Combus. 69 (2002) 249-270.

[11] D.R. Eklund, S.D. Stouffer, G.B. Northam, Study of a supersonic combustor employing swept ramp fuel injectors, J. Propul. Power 13 (1997) 697-704.

[12] Cheng-Hung Huang. Shao-pei Wang, A three-dimensional inverse heat conduction problem in estimating surface heat flux by conjugate gradient method, Int. J. Heat Mass Transfer 42 (1999) 3387-3403.

[13] J. Zhou, Y. Zhang, J.K. Chen, Z.C. Feng, Inverse estimation of surface heating condition in a finite slab with temperature-dependent thermophysical properties, Heat Transfer Eng. 32 (10) (2011) 861-875.

[14] Nazia Afrin, Z.C. Feng, Yuwen Zhang, J.K. Chen, Inverse estimation of the front surface temperature of a locally heated plate with temperature-dependent conductivity via Kirchhoff transformation, Int. J. Therm. Sci. 69 (2013) 53-60.

[15] Jianhua Zhou, Yuwen Zhang, J.K. Chen, Z.C. Feng, Inverse estimation of front surface temperature of a plate with laser heating and convention-radiation cooling, Int. J. Therm. Sci. 52 (2012) 22-30.

[16] Weiqi Qian, Kaifeng He, Yewei Gui, Qing Wang, Inverse estimation of surface heat flux for three-dimensional transient heat conduction problem, Acta Aerodyn. Sin. 28 (2) (2010) 155-161.

[17] Hongbin Gu, Lihong Chen, Wang Dan, Xinyu Chang, Lizhi, Xilong Yu, Experimental investigation on coupling characteristics of cavity flame holder and strut jet, AIAA paper 2012-5961, 2012. 